\title{
Functional Interaction of Medial Mediodorsal Thalamic Nucleus But Not Nucleus Accumbens with Amygdala and Orbital Prefrontal Cortex Is Essential for Adaptive Response Selection after Reinforcer Devaluation
}

\author{
Alicia Izquierdo and Elisabeth A. Murray \\ Section on the Neurobiology of Learning and Memory, Laboratory of Neuropsychology, National Institute of Mental Health, Bethesda, Maryland 20892-4415
}

In nonhuman primates, reward-based decision making may be assessed through choices of objects overlying two different foods, one of which has been devalued by selective satiation. The most adaptive object choices yield the food of higher value. A large body of data identifies the amygdala and orbital prefrontal cortex $(\mathrm{PFo})$ as neural mediators of adaptive responses to reinforcer devaluation. More recent work in nonhuman primates reveals the critical role of the medial, magnocellular portion of the mediodorsal nucleus of the thalamus (MDm) as well. Because both the nucleus accumbens (NA) and the MDm are anatomically related to the amygdala and PFo, and because both regions are implicated in reward processing, we tested whether either region necessarily interacts with the amygdala and PFo to mediate reinforcer devaluation effects. We used a crossed-disconnection design in which monkeys received amygdala and PFo lesions in one hemisphere combined with either NA or MDm lesions in the contralateral hemisphere. Monkeys that sustained NA disconnection, like controls, showed robust shifts in object choices in response to reinforcer devaluation. In contrast, monkeys that sustained MDm disconnection failed to adjust their object choices. Thus, MDm, but not NA, works together with the amygdala and PFo to support reward-based decision making.

\section{Introduction}

Reward-based decision making can be assessed by taxing the ability to adjust choice behavior in the face of changing values of the goal or outcome of those choices. This capacity, particularly that involving choices of objects, depends on the amygdala, the orbital prefrontal cortex (PFo), and the medial, magnocellular portion of the mediodorsal (MD) nucleus of the thalamus (MDm) (Malkova et al., 1997; Izquierdo et al., 2004; Wellman et al., 2005; Izquierdo and Murray, 2007; Machado and Bachevalier, 2007; Mitchell et al., 2007a; Rudebeck et al., 2008). We have shown previously that value-based object choices in monkeys require direct functional interaction of the amygdala and PFo (Baxter et al., 2000). Given the importance of flexible, value-based decision making in cognition, the present study explored whether functional interaction with additional brain structures makes contributions to this type of goal-directed behavior. Specifically, we assessed whether the MDm or the nucleus accumbens (NA)

Received Aug. 3, 2009; revised 0ct. 23, 2009; accepted Nov. 12, 2009.

This research was supported by the Intramural Research Program of the National Institute of Mental Health. We thank Katie Wright and Olga Sheinina for help with behavioral testing, Emily Howland for reconstructing the lesions, and Sarah Rhodes and Peter Rudebeck for comments on an earlier version of this manuscript.

Correspondence should be addressed to Dr. Elisabeth A. Murray, Laboratory of Neuropsychology, National Institute of Mental Health, Building 49, Room 1B80, 49 Convent Drive, Bethesda, MD 20892-4415. E-mail: murraye@mail.nih.gov.

A. Izquierdo's present address: Department of Psychology, California State University, Los Angeles, 5151 State University Drive, Los Angeles, CA 90032.

DOI:10.1523/JNEUROSCI.3795-09.2010

Copyright $\odot 2010$ the authors $\quad 0270-6474 / 10 / 300661-09 \$ 15.00 / 0$ works in concert with the PFo-amygdala circuit in guiding object choices in the reinforcer devaluation task.

In the primate brain, both the MDm and the NA receive dense projections from the amygdala (Aggleton and Mishkin, 1984; Fudge et al., 2002) and PFo (Goldman-Rakic and Porrino, 1985; Selemon and Goldman-Rakic, 1985; Ray and Price, 1993; Haber et al., 1995; McFarland and Haber, 2002). In addition, both regions have been implicated in reward processing. For example, $\mathrm{MDm}$ is essential for aspects of stimulus-reward learning in nonhuman primates (Aggleton and Mishkin, 1983; Gaffan and Murray, 1990; Gaffan et al., 1993; Mitchell et al., 2007a; Mitchell et al., 2007b), and similar domains are affected by larger MD thalamic lesions in rats (Hunt and Aggleton, 1998; Chudasama et al., 2001; Corbit et al., 2003). NA, in contrast, mediates both hedonic and motivational aspects of reward (Stern and Passingham, 1996; de Wit et al., 2002; Cardinal and Everitt, 2004; Pecina and Berridge, 2005). In human functional imaging studies, both NA and MDm signal anticipation of reward (Knutson et al., 2001; Galvan et al., 2005; Knutson et al., 2007; Cooper and Knutson, 2008). We have yet to understand how the MDm and NA function in concert with related brain regions when decisions are made in response to changes in reward value.

In the present study we assessed the contribution of $\mathrm{MDm}$ and NA to reward-based choice behavior by using a crosseddisconnection design. Monkeys with lesions of the PFo and amygdala in one hemisphere received excitotoxic lesions of either the MDm or NA in the contralateral hemisphere; these groups were assessed for responses to reinforcer devaluation both before 
and after the crossed disconnection. If either MDm or NA interacts critically with the amygdala and PFo in mediating object choices based on the value of the goal, then the additional lesion, which would complete the functional disconnection of these regions, would be predicted to induce a deficit. Alternatively, if interaction of the regions is not necessary for this behavior, the disconnection surgery should have little or no effect.

\section{Materials and Methods}

\section{Subjects and experimental design}

Eleven rhesus monkeys (Macaca mulatta), all male, were used. These were the same subjects studied by Izquierdo and Murray (2004). Seven monkeys received combined unilateral lesions of the amygdala and PFo in either the left or right hemisphere, and four monkeys were unoperated. (One additional monkey that had been a subject in the unilateral lesion group was unavailable for study.) All monkeys had been evaluated for discrimination learning abilities, object choices in response to reinforcer devaluation, emotional responsiveness, and food preferences (Izquierdo and Murray, 2004). Importantly, there were no differences between groups with left and right hemisphere lesions, i.e., no evidence for hemispheric specialization of function. Monkeys weighed between 7.0 and $12.8 \mathrm{~kg}$ at the beginning of the present study, were housed individually in rooms with automatically regulated lighting (light/dark 12 $\mathrm{h} / 12 \mathrm{~h}$, lights on at 7 A.M.), and were maintained on primate chow (catalog \#5038; PMI Feeds) supplemented with fresh fruit. Water was always available in the home cage.

\section{Surgical procedure}

The seven monkeys with unilateral lesions of the amygdala and PFo were randomly assigned to one of two crossed-disconnection groups destined to receive lesions of either NA or MDm, with the constraint that the side of the original removal (left or right) was balanced between groups. The NA and MDm lesions were performed an average of 26.6 months after the amygdala plus PFo lesions. Although the location and extent of the amygdala and PFo lesions have been described previously (Izquierdo and Murray, 2004), for completeness the surgical methods and histological findings will be reported below. All seven monkeys received injections of the excitotoxin ibotenic acid $(10-15 \mathrm{mg} / \mathrm{ml}$; Biosearch Technologies) in the hemisphere opposite their unilateral PFo and amygdala lesion. Four of the monkeys received injections into the NA ( $n=2$ left; $n=2$ right), and the three remaining monkeys received injections into $\operatorname{MDm}(n=2$ left; $n=1$ right) together with a partial section of the corpus callosum that enabled access to the dorsal thalamus. Of the four controls, one received a partial section of the corpus callosum to serve as a control for any effects of this procedure in the group with crossed disconnection of MDm. All the MDm and NA injections, as well as the partial callosal section in the one control, were performed between the first test of reinforcer devaluation (test 1 ) and the second (test 2). Thus, test 1 scores of the operated groups reflect the influence of unilateral lesions of the amygdala and the PFo before disconnection, whereas test 2 scores reflect the influence of the completed crossed disconnection of the amygdala and the PFo with either the MDm or the NA. For convenience, monkeys with the crossed surgical disconnection involving MDm and NA lesions are abbreviated as cases MDm- 1 to -3 and NA-1 to -4 , respectively.

At the time of surgery, anesthesia was induced with ketamine hydrochloride (10 mg/kg, i.m.) and maintained with isoflurane (1.0-3.0\%, to effect). The animals received $0.45 \%$ sodium chloride plus $5 \%$ dextrose via an intravenous drip. Aseptic procedures were used. Heart rate, respiration rate, blood pressure, expired $\mathrm{CO}_{2}$, and body temperature were monitored throughout the procedure. After the injections were completed, the wound was closed in anatomical layers.

All monkeys received a preoperative and postoperative treatment regimen consisting of dexamethasone sodium phosphate $(0.4 \mathrm{mg} / \mathrm{kg})$ and cefazolin antibiotic $(15 \mathrm{mg} / \mathrm{kg}) 1 \mathrm{~d}$ before surgery and 1 week after surgery to reduce swelling and prevent infection, respectively. At the end of surgery, and for two additional days, the monkeys received the analgesic ketoprofen (10-15 mg); ibuprofen (100 mg) was provided for five additional days.

\section{Amygdala lesion by ibotenic acid injection}

We used the same method previously described by Malkova et al. (1997) and Baxter et al. (2000). After induction of anesthesia, monkeys were placed in a stereotaxic frame. A bone flap extending over the midline was made in the appropriate portion of the cranium, and a final reading was taken on the position of the sagittal sinus, which served as the landmark for calculation of stereotaxic coordinates in the mediolateral dimension. Slits were cut in the dura to allow passage of the injection needle. Injections of ibotenic acid were placed stereotaxically throughout the amygdala in one hemisphere, with coordinates determined from magnetic resonance imaging (MRI) scans performed an average of $5.1 \mathrm{~d}$ before each surgery. Eighteen to 22 injections, each consisting of $0.6-1.0 \mu \mathrm{l}$ of ibotenic acid, were made into the amygdala via the 30 -gauge needle of a Hamilton syringe held in a micromanipulator. The injection sites were $\sim 2 \mathrm{~mm}$ apart in each plane. Each injection was made at the rate of $0.2 \mu \mathrm{l} / \mathrm{min}$, and the needle was left in place 2-3 min after each injection to limit diffusion of the toxin up the needle track. The intended lesion included the entire amygdala, including both the basolateral nuclear group as well as the central, medial, and cortical nuclei.

\section{Orbital prefrontal cortex lesion by aspiration}

For the PFo lesions, general surgical procedures were identical to those used for the amygdala lesion, except that MRI scans were not required and a regular (not stereotaxic) head holder was used. Preoperative scans were not needed because tissue removal was guided by sulcal landmarks.

A half-moon-shaped craniotomy was performed behind the orbit. The dura mater was cut near the dorsal edge of the bone opening and reflected ventrally. Using a combination of suction and electrocautery, the orbital prefrontal cortex was removed by subpial aspiration through a finegauge metal sucker, insulated except at the tip. The intended lesion extended from the fundus of the lateral orbital sulcus, which marked the lateral boundary of the lesion, to the fundus of the rostral sulcus, medially. The rostral limit of the lesion was a line joining the anterior tips of the lateral and medial orbital sulci, and the caudal limit of the lesion was $\sim 5 \mathrm{~mm}$ rostral to the junction of the frontal and temporal lobes. Thus, the lesion included Walker's areas 11, 13, and 14 and the caudal part of area 10 (Walker, 1940).

\section{NA lesion}

After induction of anesthesia, monkeys were placed in a stereotaxic frame. A large D-shaped bone flap extending over the midline was made in the appropriate portion of the cranium and a final reading was taken on the position of the sagittal sinus, which served as the landmark for the calculation of stereotaxic coordinates in the mediolateral plane. Slits were cut in the dura to allow passage of the injection needle. Injections of ibotenic acid were placed stereotaxically throughout the NA in one hemisphere, with coordinates determined from MRI scans performed an average of $4.5 \mathrm{~d}$ before each surgery. The intended lesion included both the shell and core portions of the NA. Sixteen to 18 injections, each consisting of $0.6-1.0 \mu$ l of ibotenic acid, were made via the 30 -gauge needle of a Hamilton syringe held in a micromanipulator. The injection sites were $\sim 2 \mathrm{~mm}$ apart in the anteroposterior and dorsoventral planes, and $1.5 \mathrm{~mm}$ apart in the mediolateral plane. The anteroposterior levels correspond to 23, 21, and $19 \mathrm{~mm}$ rostral to the interaural plane. Each injection was made at the rate of $0.2 \mu \mathrm{l} / \mathrm{min}$, and the needle was left in place for $2 \mathrm{~min}$ after each injection and $3 \mathrm{~min}$ after the last injection in a track to limit spread of the toxin along the needle track. The intended lesion is shown in Figure 1, and photomicrographs from case NA-3 are shown in Figure 2.

\section{MDm thalamus lesion}

After induction of anesthesia, monkeys were placed in a stereotaxic frame. A large D-shaped bone flap extending over the midline was made in the appropriate portion of the cranium and the dura mater was reflected toward the midline. To avoid puncture of large blood vessels traveling near the midline (e.g., sagittal sinus, anterior cerebral artery) we used a modified stereotaxic approach involving exposure of the dorsal thalamus. The splenium and caudal body of the corpus 


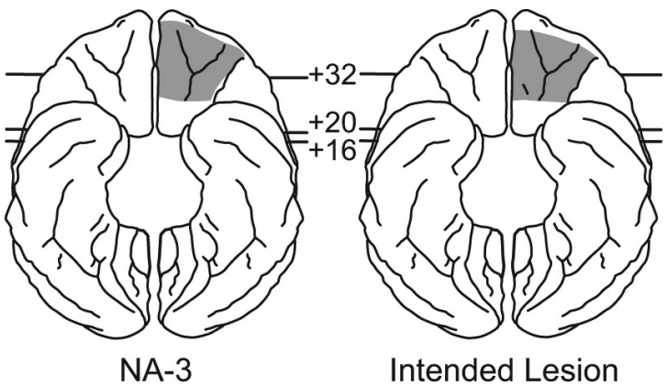

$(\mathrm{A}+\mathrm{PFO}) \times \mathrm{NA}$
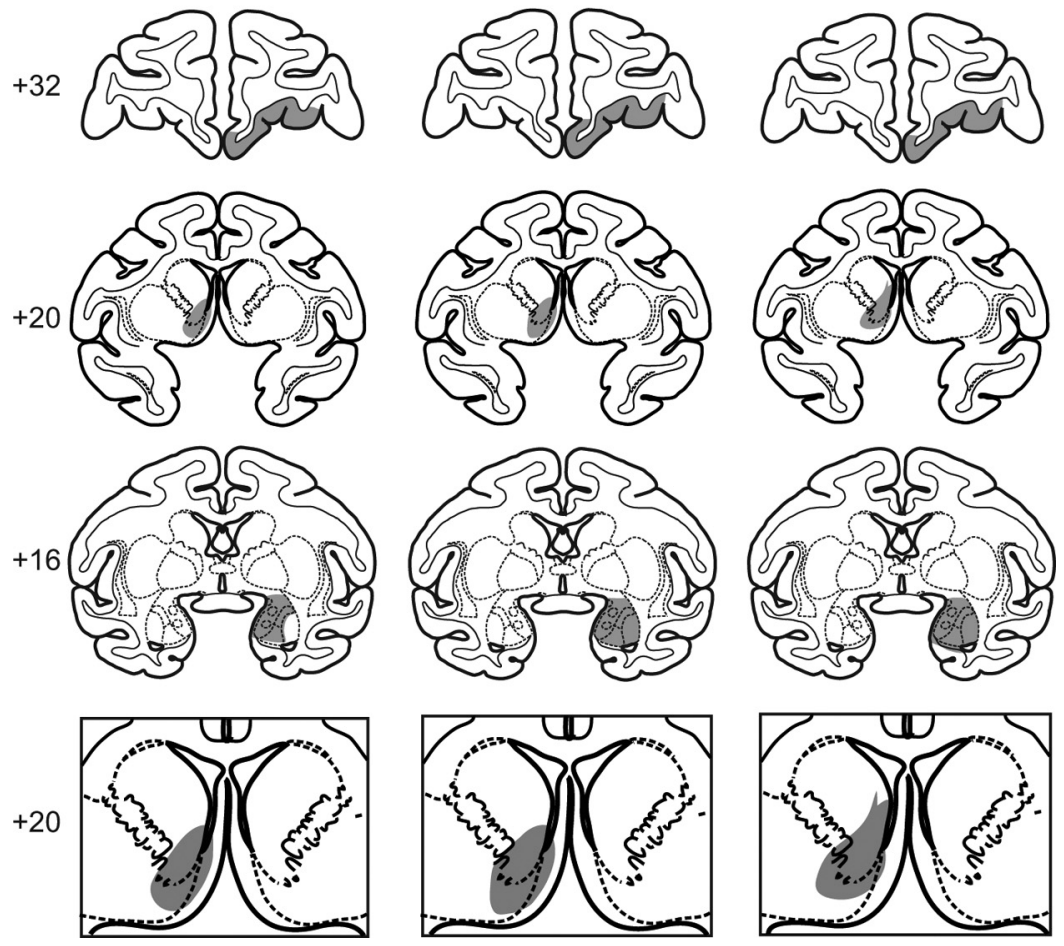

Figure 1. Representative lesion plots in monkeys with $(A+P F o) \times N A$ lesions. Middle column, Ventral view (top) and corona sections from a standard rhesus monkey brain show the intended disconnection $(A+P F 0) \times N A$ (shaded regions). Numerals indicate the distance from the interaural plane $(0)$. The intended lesion includes PFo $(+32)$ and amygdala $(+16)$ in one hemisphere and the NA in the contralateral hemisphere $(+20)$. Left column, Ventral view (top) and coronal sections show the estimated extent of the PFo, amygdala, and NA lesion in case NA-3. Right column, Ventral view (top) and coronal sections show the estimated extent of the PFo, amygdala, and NA lesion in NA-4. In the bottom row, enlarged views of the intended (middle) and estimated lesions for NA-3 (left) and NA-4 (right) are also shown.

callosum were sectioned to reveal the dorsal surface of the thalamus. Using the posterior commissure and habenula as landmarks, the location of the MDm thalamus was estimated and injections of ibotenic acid were placed via the 30 -gauge needle of a Hamilton syringe held in a micromanipulator. Specifically, each monkey received a total of 11 injections of ibotenic acid, $1.0 \mu \mathrm{l}$ per site, placed in four separate needle penetrations. The sites were distributed at three anteroposterior levels: caudal series, $1.0 \mathrm{~mm}$ rostral to the habenula, $1.4 \mathrm{~mm}$ from the thalamic midline, 2.5 and $4.5 \mathrm{~mm}$ ventral to the surface of the thalamus; middle series, $2.5 \mathrm{~mm}$ rostral to the habenula, 1.4 and 2.9 $\mathrm{mm}$ from the midline, $2.0,3.5$, and $5.0 \mathrm{~mm}$ from the surface of the thalamus; and anterior series, $4.0 \mathrm{~mm}$ rostral to the habenula, $1.4 \mathrm{~mm}$ from the midline, and 2.0, 3.6, and $5.2 \mathrm{~mm}$ from the surface of the thalamus. The three anteroposterior levels correspond to 5.0, 6.5, and 8.0 $\mathrm{mm}$ rostral to the interaural plane. One monkey received each injection as a bolus and the other two received injections made at the rate of $0.2 \mu \mathrm{l} / \mathrm{min}$. After each injection was completed, the needle was left in place $2-3 \mathrm{~min}$ to limit diffusion of the toxin up the needle track. The intended lesion is shown in Figure 3 and photomicrographs from case MDm-1 are shown in Figure 4.
Control surgery

In one control monkey (CON-4), the splenium and the caudal body of the corpus callosum were sectioned in the same manner as that in monkeys that received injections of excitotoxin in the MDm. This was intended to serve as a control for the possibility that a partial section of the corpus callosum, which was not performed in the monkeys receiving NA injections, might disrupt reinforcer devaluation effects.

\section{Assessment of the lesions}

At the completion of the experiment, the monkeys were anesthetized with ketamine, given a lethal dose of sodium pentobarbital $(100 \mathrm{mg} /$ kg, i.p.), and transcardially perfused with $0.9 \%$ saline followed by a solution of aldehyde fixatives. The brains were removed from the cranium, photographed, and cryoprotected in a solution of $20 \%$ glycerol and $10 \%$ formalin. Tissue was sectioned in the coronal plane at $50 \mu \mathrm{m}$ on a freezing microtome. Every fifth section was mounted on gelatin-coated slides, defatted, stained with thionin, and coverslipped. The lesions were plotted onto drawings of coronal brain sections of a standard rhesus monkey brain.

\section{PFo and amygdala lesions}

Because we first studied the effects on behavior of combined unilateral PFo and amygdala lesions, the extent of the lesions was initially evaluated using postoperative MR scans (Izquierdo and Murray, 2004). For present purposes, the extent of PFo and amygdala damage has been reassessed through microscopic examination of tissue after traditional histological processing of the brains. The PFo and amygdala lesions were generally as intended. Whereas the PFo lesions were complete or nearly so in six of the seven operated monkeys, the extent of the amygdala lesions was more variable. Specifically, although the (unilateral) amygdala damage was extensive in all monkeys, there was partial sparing of either the lateral nucleus (NA-1, NA-3) or the ventromedial amygdala, including portions of the medial basal and basal accessory nuclei (MDm-2, MDm-3, NA-2). Importantly, there was no systematic difference in the extent or location of either PFo or amygdala damage in the groups that received the MDm and NA lesions. Gliosis, presumably a result of retrograde degeneration secondary to the PFo lesion (Rose and Woolsey, 1943), was visible in the same hemisphere as the PFo lesion in the MDm thalamus in all monkeys in both experimental groups. Not surprisingly, the gliosis roughly mirrored the location and extent of the MDm lesions in MDm-1 to MDm-3 (see below).

Inadvertent damage associated with the PFo and amygdala lesions, again unilateral, was limited to $1-2 \mathrm{~mm}$ of the rostral hippocampus (NA-2, NA-3, MDm-2, MDm-3), ventral claustrum (MDm-1, MDm-3), the piriform cortex $(\mathrm{MDm}-1)$, and the lower bank of the principal sulcus, the latter apparently due to infarction (NA-2).

\section{NA lesions}

All four monkeys that received ibotenate injections in the ventral striatum sustained extensive (unilateral) damage to the nucleus accumbens, as intended (see Fig. 1). None of the lesions could be considered complete. In NA-1 there was sparing of the most ventrolateral portion of the NA at anterior levels. NA-2 and NA-3 sustained the most extensive lesions. In these cases, the lesion affected the full anteroposterior extent of the NA; inadvertent damage in both cases involved small portions of the 
adjacent medial frontal cortex (area 25) and the vertical limb of the diagonal band. The lesion in NA-4 was centered slightly dorsal to the ideal position; as a result, the lesion encroached on the caudate nucleus and spared the most ventral portion of the NA.

\section{MDm lesions}

All three subjects that received ibotenate injections in the medial thalamus sustained extensive (unilateral) damage to the magnocellular division of the mediodorsal nucleus of the thalamus, as intended (see Fig. 3). Cell loss and gliosis extended for 2.5 (MDm-2), 3.5 (MDm$3)$, and $4.0(\mathrm{MDm}-1) \mathrm{mm}$ in anteroposterior extent (between +4.5 and $+8.5 \mathrm{~mm}$ rostral to the interaural plane) and affected the full dorsoventral extent of MDm. As a result, the damage to MDm was substantial in MDm-2 and nearly complete in the two others. The lesion in MDm-2 encroached on the midline, and the lesion in $\mathrm{MDm}-1$ was centered slightly further from the midline than that in either MDm-2 or MDm-3. All three monkeys also sustained transection of the splenium and the caudal body of the corpus callosum. Two of the three monkeys (MDm-2, MDm-3) also sustained partial damage to the fornix in the hemisphere with the MDm injections.

\section{Control surgery}

As intended, the splenium and caudal body of the corpus callosum were sectioned in case CON-4.

\section{Apparatus and materials}

Monkeys were trained in a modified Wisconsin General Testing Apparatus (WGTA). The WGTA consists of a large enclosed compartment that holds the test cage plus monkey, together with a smaller enclosed compartment that contains the test tray. The test compartment was illuminated with two $60 \mathrm{~W}$ bulbs, whereas the monkey's compartment was unlit. During test sessions, the room was unlit as well. An opaque screen separated the monkey compartment from the test compartment during intertrial intervals. In addition, a one-way viewing screen, located between the experimenter and the test compartment, allowed the experimenter to view the monkey's responses during trials without being seen by the monkey. The test tray, measuring $19.2 \mathrm{~cm}$ (width) $\times 72.7 \mathrm{~cm}$ (length) $\times 1.9 \mathrm{~cm}$ (height), contained two food wells $290 \mathrm{~mm}$ apart, center to center, on the midline of the tray. The wells were $38 \mathrm{~mm}$ in diameter and $6 \mathrm{~mm}$ deep.

Three "junk" objects were used for pretraining only. For the main experiment, a novel set of 120 junk objects that varied widely in color, shape, and size was used. Food rewards for each monkey were two of the following: a half peanut, a fruit snack (Giant Food), or a chocolate candy (M\&Ms; Mars). The same two foods that had been used for each monkey in the earlier study of reinforcer devaluation (Izquierdo and Murray, 2004) were assigned to be used again.

\section{Testing procedure}

Pretraining. Monkeys were required to displace one of the three objects dedicated to this phase to retrieve a food reward hidden in a well underneath. Objects were presented in random order, one at a time, and always covered a baited well. Before proceeding to the next stage, each monkey was required to complete a single session of 50 trials.

Visual discrimination learning. Monkeys were trained to discriminate 60 pairs of objects. The 120 objects were assigned to 60 fixed pairs. For each pair, one object was arbitrarily designated as positive (i.e., $\mathrm{S}+$, baited with a food reward) and the other negative (i.e., S-, unbaited). Half of the positive objects were assigned to be baited with food 1 , and the
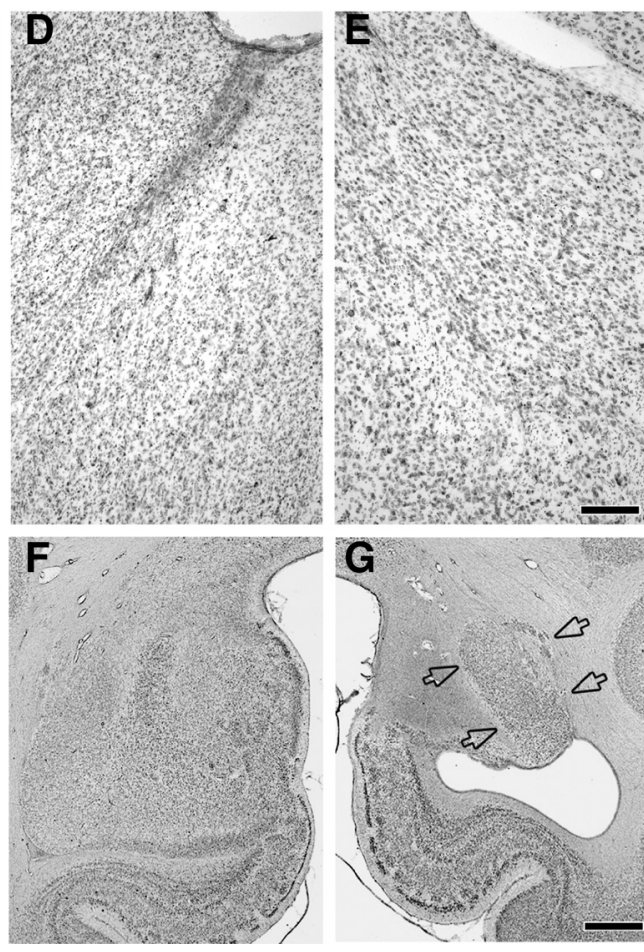

Figure 2. Photomicrographs of Niss-stained coronal sections through the lesion in NA-3. A, Filled arrows mark the boundaries of the orbital prefrontal cortex lesion. $\boldsymbol{B}$, Rectangles outline small portions of the left and right NA shown at a higher magnification

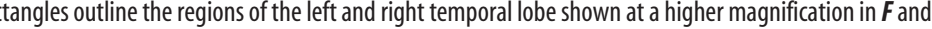
excioxic amygdala lesion (G). Note the extensive cell loss and gliosis in the basal, basal accessory, central, medial, and cortical nuclei of the amygdala but the partial sparing of the lateral nucleus of the amygdala (open arrows). Scale bars, (in $E) D, E, 200 \mu \mathrm{m}$; (in $\boldsymbol{G}), F, G, 2 \mathrm{~mm}$.

other half with food 2. On the first trial, the two objects comprising pair one were presented for choice, each overlying one of the two food wells on the test tray. If the monkey displaced the $\mathrm{S}+$, it was allowed to take the food reward hidden underneath. If the monkey chose the $S-$, the trial was terminated without correction. After a $20 \mathrm{~s}$ intertrial interval, this procedure was repeated with the next pair of objects, and so on, until all 60 pairs had been presented. The positive and negative assignment of objects, the presentation order of the object pairs, and the food reward assignments remained constant across sessions; the left-right position of positive objects followed a pseudorandom (Gellerman) order. Monkeys were tested at the rate of one session per day for $5 \mathrm{~d}$ per week. Criterion was set at a mean of $90 \%$ correct over five consecutive days (i.e., 270 correct responses in 300 trials).

Reinforcer devaluation test 1 . After the monkeys had acquired the discrimination problems, their choices of objects were assessed in four critical test sessions. In these sessions, only the positive $S+$ objects were used. Thirty pairs of objects, each consisting of one food 1 object and one food 2 object, were presented to the monkey for choice. Both objects were baited with the same foods used during acquisition. In each trial, the monkeys were allowed to choose only one of the objects in each pair to obtain the food reward hidden underneath, and the choice was scored by the experimenter. Two of the four critical test sessions were preceded by a selective satiation procedure intended to devalue one of the two foods, and the other two sessions were preceded by no satiation procedure and provided baseline measures. At least $2 \mathrm{~d}$ of rest followed each session that was preceded by selective satiation. In addition, between critical test sessions the monkeys were given one regular training session with the original 60 pairs of objects presented in the same manner as during initial learning. This procedure ensured that any long-lasting effects of selective satiation that might affect the outcome of later critical test sessions could be detected. Objects were paired anew for each critical session, and ses- 


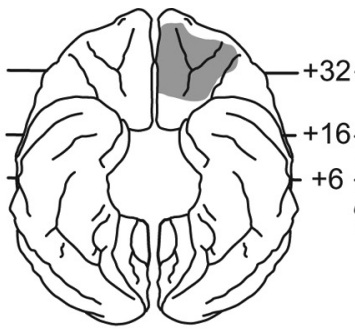

MDm-1
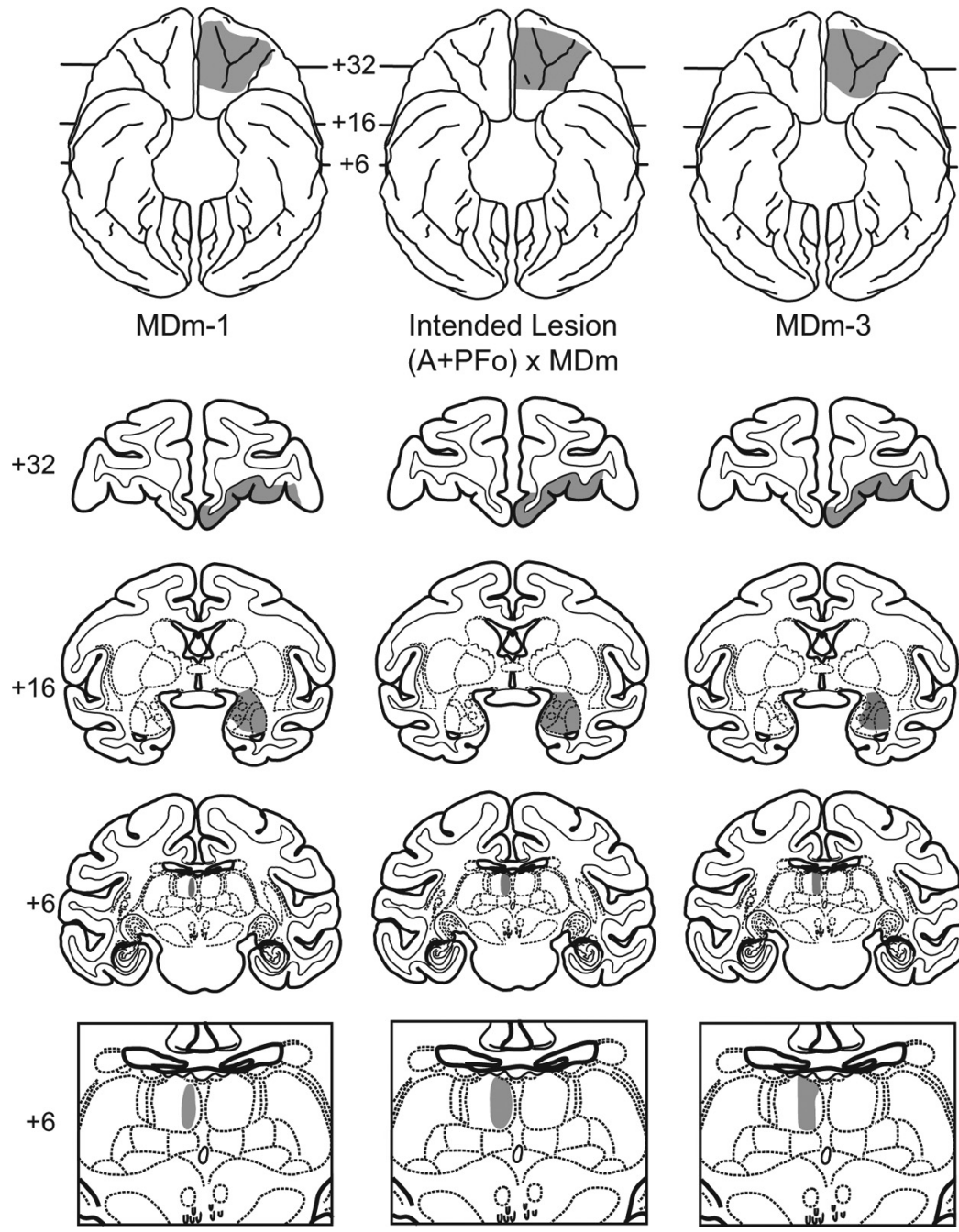

MDm-3

Intended Lesion
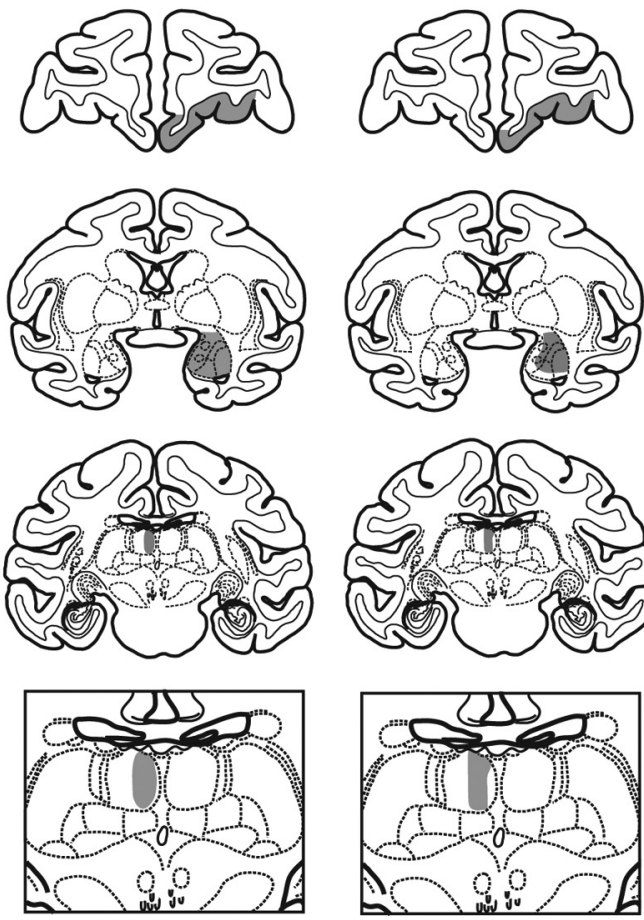

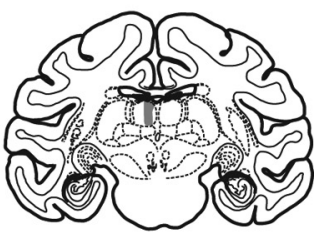

Figure 3. Representative lesion plots in monkeys with $(A+P F o) \times M D m$ lesions. In the middle column a ventral view (top) and coronal sections from a standard rhesus monkey brain show the intended disconnection ( $\left.A+P F_{0}\right) \times M D m$ (shaded regions). Numerals indicate distance from the interaural plane $(0)$. The intended lesion includes PFo $(+32)$ and amygdala $(+16)$ in one hemisphere and the MDm thalamus in the contralateral hemisphere $(+6)$. Left column, Ventral view (top) and coronal sections show the estimated extent of the PFo, amygdala, and MDm lesion in case MDm-1. Right column, Ventral view (top) and coronal sections show the estimated extent of the PFo, amygdala, and MDm lesion in MDm-3. Bottom row, Enlarged views of the intended (middle) and estimated lesions for MDm-1 (left) and MDm-3 (right) are also shown.

sions were administered in the following order for each monkey: (1) first baseline session; (2) session preceded by selective satiation with food 1 ; (3) second baseline session; (4) session preceded by selective satiation with food 2. The unit of analysis, the "difference score," is defined as the change in choices of object type (food 1- and food 2-associated objects) in the sessions preceded by selective satiation as compared with the mean of the two baseline sessions. The difference scores derived from each of the two critical sessions preceded by selective satiation were calculated separately and then summed to provide the final difference score for each test.

Selective satiation. For the selective satiation procedure, a container measuring $7.7 \mathrm{~cm}$ (width) $\times 10.3 \mathrm{~cm}$ (length) $\times 7.7 \mathrm{~cm}$ (height) and attached to the monkey's home cage was filled with a known quantity of either food 1 or food 2 while the monkey was in its home cage. The monkey was then left to eat unobserved for $15 \mathrm{~min}$, at which point the experimenter checked to see whether it had eaten all the food. If it had, the food container was refilled. Whether additional food was given or not, the experimenter started observing the monkey through a window outside the animal housing room $30 \mathrm{~min}$ after initiation of the satiation procedure. Observation continued until the monkey failed to take food for five consecutive minutes. If the monkey consumed all the available food again, the container was refilled and observation continued until the monkey refrained from taking food for $5 \mathrm{~min}$. When this criterion was met, the test session in the WGTA was initiated within $10 \mathrm{~min}$. For baseline sessions, the monkey was simply taken from its home cage to the WGTA without undergoing a selective satiation procedure. For each instance of selective satiation, the amount of food eaten and the elapsed time spent in the satiation procedure were noted. At the end of the satiation procedure, any food remaining in the food container or dropped on the floor of the cage was taken into account when estimating the total amount of food eaten.

Reinforcer devaluation test 2 . After surgery or a period of rest for the three unoperated controls, the monkeys were retrained on the original set of object discrimination problems in the same manner and with the same criterion as before. After relearning was completed, the reinforcer devaluation test was repeated in exactly the same manner as before.

Reinforcer devaluation test 3 (food choices). Test 3 was administered following test 2 , with $2 \mathrm{~d}$ of rest intervening. Test 3 was executed in the same manner as tests 1 and 2 with the exception that the critical trials were administered without objects overlying the foods. That is, monkeys were required to make visual choices of the foods. This procedure allowed us to determine whether satiety transferred from the home cage to the test apparatus, as we assumed. In addition, the test provided confirmation that the monkeys were able to visually identify the two foods and discriminate between them.

\section{Results}

\section{Visual discrimination: initial learning}

Trials and errors to criterion (i.e., all trials and errors accrued in all sessions up to but not including the criterion run) are provided in Table 1. Monkeys with unilateral lesions scored fewer errors in learning the 60 object pairs than did unoperated controls $\left(t_{(9)}=2.6 ; p=0.03\right)$. In addition, an ANOVA with repeated measures on the percentage of correct responses obtained in the first eight sessions of learning (the only sessions common to all monkeys) showed faster acquisition for monkeys with unilateral lesions relative to controls (interaction of group by stage: $F_{(7,63)}=20.4 ; p<0.01$ ). During this phase, it became apparent that two of the four controls (CON-2 and CON-4) were disinclined to accept one of the two food rewards. Once a different, more preferred food was substituted for the disliked one, learning proceeded rapidly. It is likely that the inadvertent use of a nonpreferred food in these two monkeys accounts for the learning difference between groups. Despite this group difference in the rate of learning the 60 pairs, an ANOVA revealed that the two groups destined to receive the NA and MDm lesions did not differ from each other on errors to criterion (all $p$ values $>0.70$ ).

\section{Visual discrimination: relearning}

The numbers of trials and errors required to relearn the 60 object pairs are provided in Table 1. Because trials and errors scored 
during the $5 \mathrm{~d}$ criterion run are not included in our tally, relearning scores of zero (0) reflect perfect retention of the preoperatively learned discrimination pairs. After the surgical disconnection was completed (or control surgery or rest for controls), monkeys quickly relearned the 60 object pairs. An ANOVA on errors to reattain criterion revealed no significant differences between groups $\left(F_{(2,8)}=0.88\right.$; $p=0.45)$.

\section{Reinforcer devaluation}

\section{Difference scores}

As explained earlier, our main dependent measure is the difference score, which is the change in choices of object type (food 1- and food 2-associated objects) in the sessions preceded by selective satiation as compared with baseline sessions. Positive difference scores indicate sensitivity to reinforcer devaluation. On test 1 , which took place before the crosseddisconnection surgery, all three groups showed robust responses to reinforcer devaluation (see Fig. 5). On test 2, after the disconnection, the difference scores of the group with the added MDm lesion were reduced whereas the scores of monkeys in the other two groups increased on average.

A $2 \times 3$ ANOVA with repeated measures on the difference scores using a within-subjects factor of test (reinforcer devaluation tests 1 and $2)$ and a between-subjects factor of group [ $(\mathrm{A}+\mathrm{PFo}) \times \mathrm{MDm}$, $(\mathrm{A}+\mathrm{PFo}) \times \mathrm{NA}, \mathrm{CON}$; where $\mathrm{A}$ is amygdala and $\mathrm{CON}$ is control] revealed a main effect of group $\left(F_{(2,8)}=12.37 ; p=0.004\right)$ and a significant interaction between group and test $\left(F_{(2,8)}=\right.$ 5.15; $p=0.04)$. Results from post hoc ANOVAs showed that the groups did not differ on test $1\left(F_{(2,8)}=1.15 ; p=0.36\right)$, indicating that all monkeys performed comparably at the beginning of the experiment. However, the groups differed significantly on test 2 $\left(F_{(2,8)}=26.15 ; p<0.001\right)$ [Fisher's PLSD, group CON scores $=$ $\operatorname{group}(\mathrm{A}+\mathrm{PFo}) \times \mathrm{NA}>\operatorname{group}(\mathrm{A}+\mathrm{PFo}) \times \mathrm{MDm}]$.

On test 3, which involved choices between the two foods, the three groups showed robust responses to reinforcer devaluation. When given the choice between a sated and a nonsated food, all monkeys avoided the sated food on virtually every trial, thereby earning a high difference score (mean difference scores: CON, 29.5; $\left.(\mathrm{A}+\mathrm{PFo}) \times \mathrm{NA}, 29.5 ;\left(F_{(2,8)}\right) \times \mathrm{MDm}, 29.3\right)$. An ANOVA confirmed that the groups did not differ on this measure $\left(F_{(2,8)}=\right.$ $0.06 ; p=0.96)$.

\section{Trial-by-trial choice during critical test sessions}

To determine whether learning (or other factors) might be influencing choices of object type (food 1 and food 2 associated objects) within critical sessions, we conducted a more fine-grained analysis of the data. First, for each monkey, each trial of the 30 trial sessions that followed selective satiation was scored systematically with a 1 to denote the choice of the object associated with the food not devalued through satiation or with a 0 (zero) to denote the choice of the object associated with the devalued food. Data for the two sessions that followed satiation were averaged
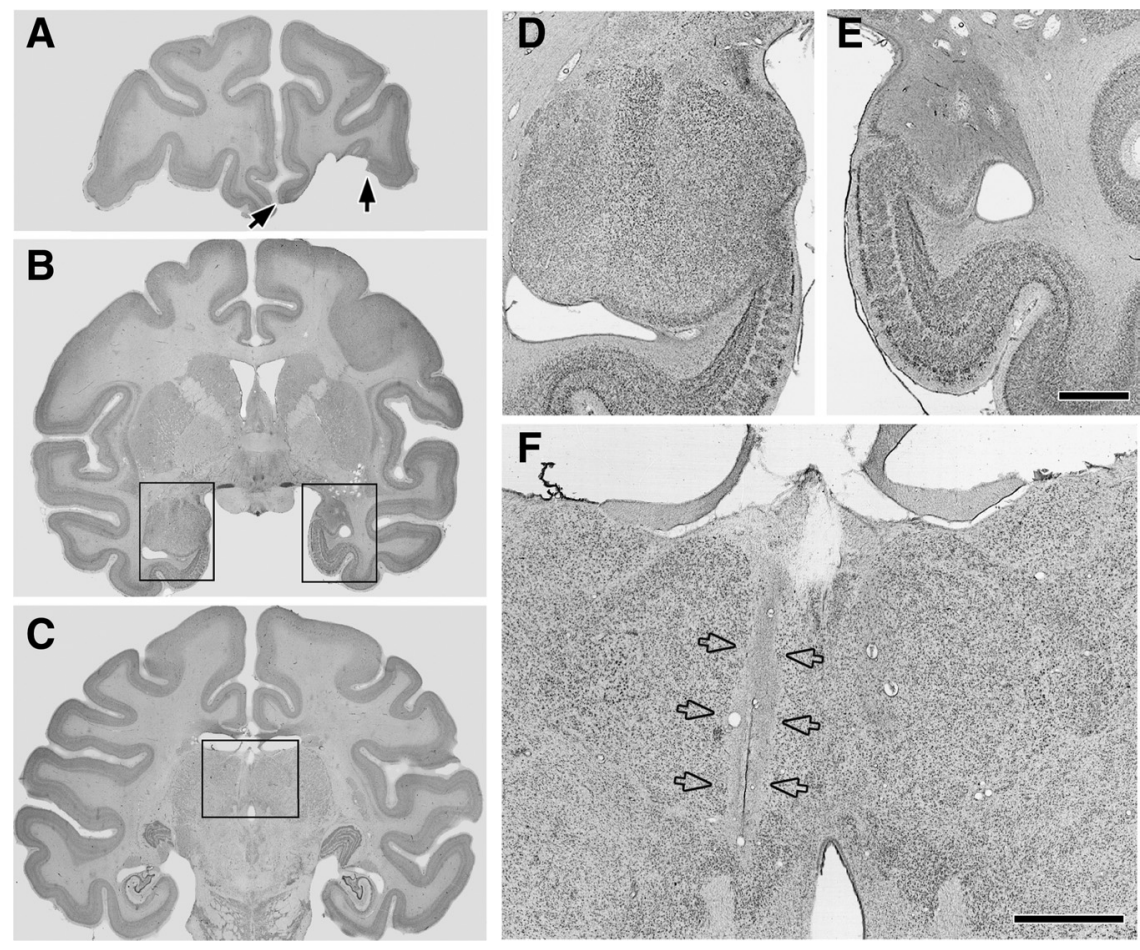

Figure 4. Photomicrographs of Nissl-stained coronal sections through the lesion in MDm-1. A, Filled arrows mark the boundaries of the orbital prefrontal cortex lesion. $\boldsymbol{B}$, Rectangles outline the regions of the left and right temporal lobe shown at a highe ind $\boldsymbol{E}$. C, The rectangle outlines the portion of the thalamus shown at higher magnification in $F$. D, E, Photomi-

and then collapsed into five-trial blocks. Group mean scores are provided in Figure 6; the higher the score, the more adaptive the response, a measure we refer to here as "adaptive choice." A comparison of monkeys' adaptive choices for both test 1 and test 2 was conducted using ANOVA with repeated measures on five-trial blocks. As expected, on test 1, before the surgical disconnection there was no significant effect of group $\left(F_{(2,19)}=1.26\right.$; $p=0.31)$, trial block $\left(F_{(5,95)}=1.73 ; p=0.13\right)$, or group $\times$ trial block interaction $\left(F_{(10,95)}=0.95 ; p=0.49\right)$. On test 2 , after disconnection of either NA or MDm, there was a robust effect of group $\left(F_{(2,19)}=11.59 ; p<0.001\right)$ and group $\times$ trial block interaction $\left(F_{(5,95)}=2.94 ; p<0.001\right)$, but no significant withinsubject effect of trial block $\left(F_{(5,95)}=0.56 ; p=0.73\right)$. Post hoc Fisher's PLSD revealed no significant differences between groups $(\mathrm{A}+\mathrm{PFo}) \times \mathrm{NA}$ and CON. For all but the first five-trial block, group $(\mathrm{A}+\mathrm{PFo}) \times \mathrm{MD}$ differed significantly from CON (all $p$ values $<0.03)$ and group $(\mathrm{A}+\mathrm{PFo}) \times \mathrm{NA}($ all $p$ values $\leq 0.06)$.

\section{Amount of food consumed during selective satiation}

The amounts consumed in the satiation procedures were analyzed using an ANOVA with repeated measures on the three tests and did not differ by group $\left(F_{(2,8)}=1.53 ; p=0.27\right)$. There was similarly no significant within-subjects effect of test $\left(F_{(2,16)}=1.85 ; p=0.19\right)$ or test by group interaction $\left(F_{(4,16)}=\right.$ $0.97 ; p=0.45)$.

\section{Discussion}

We found that macaque monkeys with a surgical disconnection of the MDm from the amygdala and the PFo failed to adjust their object choices in the face of changes in the value of the goal. In contrast, surgical disconnection of the NA from the amygdala 
Table 1. Trials and errors to criterion

\begin{tabular}{|c|c|c|c|c|}
\hline & \multicolumn{2}{|l|}{ Learning } & \multicolumn{2}{|c|}{ Relearning } \\
\hline & $\mathrm{T}$ & $E$ & $\mathrm{~T}$ & $E$ \\
\hline MDm-1 & 360 & 98 & 0 & 0 \\
\hline MDm-2 & 180 & 72 & 0 & 0 \\
\hline MDm-3 & 420 & 117 & 120 & 25 \\
\hline Mean & 320.0 & 95.6 & 40.0 & 8.3 \\
\hline NA-1 & 240 & 87 & 0 & 0 \\
\hline NA-2 & 600 & 166 & 240 & 46 \\
\hline NA-3 & 480 & 166 & 0 & 0 \\
\hline NA-4 & 540 & 135 & 120 & 17 \\
\hline Mean & 465.0 & 138.5 & 90.0 & 15.8 \\
\hline CON-1 & 360 & 116 & 0 & 0 \\
\hline $\mathrm{CON}-2$ & 2160 & 489 & 420 & 73 \\
\hline $\mathrm{CON}-3$ & 720 & 182 & 0 & 0 \\
\hline $\mathrm{CON}-4^{*}$ & 1980 & 585 & 480 & 75 \\
\hline Mean & 1305.0 & 343.0 & 225.0 & 37.0 \\
\hline
\end{tabular}

Trials $(T)$ and errors (E) to acquire 60 visual object discrimination problems before surgery (learning) that completed a crossed surgical disconnection of the amygdala plus PFo with either the medial portion of the MDm or the NA and to relearn the same problems after the disconnection had been completed (relearning). Prior to the present study, all monkeys in the two experimental groups had sustained unilateral lesions of the amygdala and PFo in one hemisphere (left or right). MDm-1 to MDm-3: monkeys designated to receive crossed disconnection of the amygdala plus $\mathrm{PF} \times \mathrm{MDm}$; NA-1 to NA-4: monkeys designated to receive crossed disconnection of the amygdala plus $\mathrm{PF} 0 \times \mathrm{NA}$ CON-1 to CON-3: unoperated controls; $\mathrm{CON}-4^{*}$ : operated control with partial section of the corpus callosum.

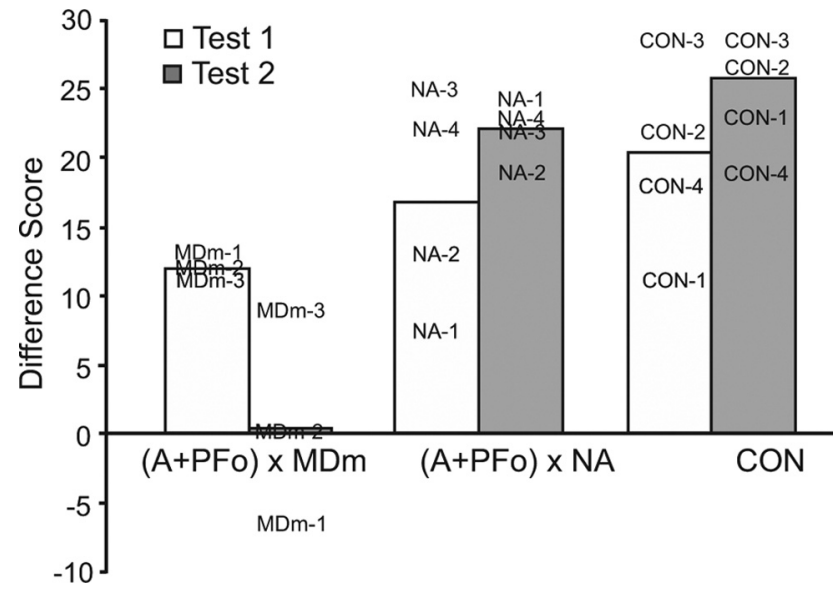

Figure 5. Difference scores on the reinforcer devaluation task (test 1 and test 2). Bars represent mean difference scores of monkeys with disconnections of $A+P F o$ from MDm (MDm-1 to MDm-3), disconnections of A + PFo from NA (NA-1 to NA-4), and controls. CON-1 to CON-3 remained unoperated; $\mathrm{CON}-4$ sustained a lesion of the splenium and caudal body of the corpus callosum. The higher the score, the greater the response to changes in reinforcer value. At the time of test 1, monkeys in the groups destined to receive surgical disconnection had sustained unilateral $A+P F o$ lesions whereas at the time of test 2 they had sustained the completed surgical disconnection, either $\left(A+P F_{0}\right) \times M D m$ or $\left(A+P F_{0}\right) \times N A$. The case numbers represent scores for individual monkeys.

and the PFo produced no such impairment. Thus, the MDm but not the NA is a constituent of the circuitry guiding flexible, goaldirected object choices, and MDm must functionally interact with the amygdala and PFo in mediating such behavior.

Although our monkeys with combined unilateral lesions of amygdala and PFo were impaired on the reinforcer devaluation task immediately after surgery (Izquierdo et al., 2004), they did not differ from the controls on test 1 of the present study, which was performed $\sim 24$ months later (Fig. 5, test 1 ). Thus, unlike groups with bilaterally symmetrical lesions of PFo (Izquierdo et al., 2004) and amygdala (Izquierdo and Murray, 2007), both of which exhibited impairments $\sim 19$ months following surgery, the group of monkeys with unilateral amygdala plus PFo lesions within one hemisphere showed recovery of function.
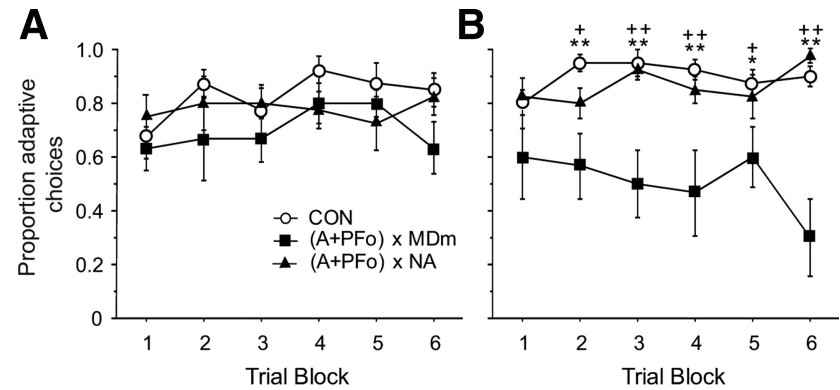

Figure 6. Trial-by-trial object choices on critical test sessions after selective satiation. $A$, Curves show mean proportion of adaptive choices of controls ( $C O N)$ and monkeys with unilateral A + PFo lesions on test 1 . Note that at the time of test 1 the experimental groups are destined to receive crossed-disconnection surgery but have not yet received it. $\boldsymbol{B}$, Mean proportion of adaptive choices of monkeys with surgical disconnection of $A+P F o$ from MDm, $\left(A+P F_{0}\right) \times M D m$, or disconnection of $A+P F$ from NA, $\left(A+P F_{0}\right) \times N A$, and controls on test 2 . The higher the score, the more adaptive the response to changes in reinforcer value. Group $(\mathrm{A}+\mathrm{PF} 0) \times \mathrm{MDm}$ significantly different from $\mathrm{CON} ;{ }^{*} p<0.05 ;{ }^{* *} p<0.01$. Group $\left(A+P F_{0}\right) \times M D m$ significantly different from $(A+P F 0) \times N A ;+p \leq 0.06 ;++p<0.01$.

\section{Methodological considerations}

Monkeys with selective, excitotoxic lesions of the MDm thalamus in one hemisphere and combined lesions of the amygdala and $\mathrm{PFo}$ in the other hemisphere [group $(\mathrm{A}+\mathrm{PFo}) \times \mathrm{MDm}$ ] showed intact retention of preoperatively learned object pairs and normal amounts of food consumption during satiation procedures. In addition, when given pairwise choices between sated and nonsated foods, monkeys with crossed disconnection of MDm, like controls, avoided selection of the sated food. Furthermore, all monkeys showed distinct and stable food preferences, as evidenced from their baseline choices of the two foods (baseline, test 3). Accordingly we can rule out changes in visual perceptual abilities, satiety mechanisms, and food preferences as the source of the impairment in the group with MDm lesions. Instead, the impairment must be either in linking objects with the current value of the food or in using this information to guide response selection, or both.

Transection of the splenium and caudal body of the corpus callosum, which was also performed in this group, seems unlikely to account for the impairment; the one operated control monkey receiving this procedure $(\mathrm{CON}-4)$ obtained a difference score comparable to that of intact controls on tests 2 and 3 . With the exception of the projection from PFo to MDm, and of the amygdala to $\mathrm{MDm}$, there are few crossed projections among the amygdala, MDm, and PFo (Preuss and Goldman-Rakic, 1987). Although there is a substantial bilateral projection from PFo to MDm, the massa intermedia is evidently the major route of decussation of these fibers (Preuss and Goldman-Rakic, 1987; see p. 274). In addition, these authors explicitly state that few, if any, crossed prefrontal-thalamic fibers travel in the large forebrain commissures (Preuss and Goldman-Rakic, 1987; see p. 275). Furthermore, projections from the amygdala to MDm travel largely via routes other than the forebrain commissures (Aggleton and Mishkin, 1984). Nevertheless, it is difficult to rule out the possibility that some crossed fibers traveling in the forebrain commissures might have been severed in the group with MDm lesions, which received the partial section of the corpus callosum, and that this contributed to their deficit. As for NA, again there appear to be few crossed projections between the NA and PFo or amygdala. For example, Friedman et al. (2002) reported a complete lack of crossed projections from the amygdala to NA. Because no partial callosal section was performed in the group with 
NA lesions, we cannot rule out the possibility that the addition of this procedure, had we carried it out, might have yielded an impairment in this group. Finally, partial damage to the fornix, either alone or in combination with the added MDm lesion, seems unlikely to account for the impairment; the one monkey with little or no damage to the fornix (MDm-1) displayed the largest drop in difference scores after the surgery.

\section{Neural circuitry underlying responses to reinforcer devaluation}

In macaque monkeys, the amygdala (Malkova et al., 1997; Wellman et al., 2005; Izquierdo and Murray, 2007; Machado and Bachevalier, 2007), PFo (Izquierdo et al., 2004; Machado and Bachevalier, 2007), and MDm (Mitchell et al., 2007a) are known to be essential for reward-guided decision making as evaluated by the reinforcer devaluation task. Similar results have been found in rats (Hatfield et al., 1996; Gallagher et al., 1999; Ostlund and Balleine, 2008). In addition, it has been shown that the amygdala must functionally interact with PFo to guide object choices in this task (Baxter et al., 2000). The present study extends these findings by showing that the amygdala, $\mathrm{PFo}$, and MDm functionally interact in mediating this behavior.

The selective contributions of these three regions to mediating responses to reinforcer devaluation is not fully understood. Although the amygdala, particularly the basolateral amygdala, has been implicated in updating previously established representations of reinforcer value during satiation (Wang et al., 2005; Wellman et al., 2005), recent findings suggest instead that the amygdala may be essential for the formation of associations between cues (or responses) and detailed sensory properties of reinforcers, an ability that is taxed when multiple stimulusoutcome relationships are learned (Ostlund and Balleine, 2008; Johnson et al., 2009).

Several studies implicate the PFo in representing the value of expected outcomes (Holland and Gallagher, 2004; Burke et al., 2008). In accord with these suggestions, behavioral neurophysiology studies in macaques indicate that at the time monkeys are viewing images, neurons in PFo are signaling the value of rewards that have been associated with those images (Wallis and Miller, 2003; Padoa-Schioppa and Assad, 2006; Kennerley et al., 2008). PFo appears to be an essential site of storage of representations of expected outcomes after changes in the incentive value of the reinforcer (Pickens et al., 2003).

As indicated in the Introduction, MDm has been implicated in reward-guided response selection, although some have noted a role for this region in shifting between behavior-guiding rules or strategies (Block et al., 2007; Pickens, 2008), and evidence suggests a specialization for acquisition as opposed to retrieval (monkeys: (Mitchell et al., 2007b; Mitchell and Gaffan, 2008); rats: (Hunt and Aggleton, 1991; Neave et al., 1993; Hunt and Aggleton, 1998; cf. Floresco et al., 1999). In rats, MD lesions encompassing the central, medial, and lateral nuclei of the MD thalamus result in disrupted cue-guided response selection (Corbit et al., 2003; Ostlund and Balleine, 2008). The contribution of the MD thalamus to mediating reinforcer devaluation effects appears to be distinct from that of other subregions of the thalamus (Corbit et al., 2003) and may be quite distinct from the role of the amygdala in reward processing. Whereas the amygdala and the MD thalamus each contribute to both instrumental and Pavlovian-based processes, the MD thalamus seems to have a more circumscribed role in stimulus-guided selection of instrumental actions (Ostlund and Balleine, 2008). Our task does not allow us to distinguish between Pavlovian and instrumental contributions to performance. Accordingly, future studies in mon- keys should aim to dissociate Pavlovian and instrumental components of this task.

NA has been implicated in both hedonic and motivational aspects of reward (Stern and Passingham, 1996; de Wit et al., 2002; Cardinal and Everitt, 2004; Pecina and Berridge, 2005). Although NA is apparently not necessary for ascribing and responding to the representations of instrumental outcomes or goals (de Wit et al., 2002), it may play a role in Pavlovian processes whereby appetitive outcomes stimulate the vigor of instrumental performance (de Wit et al., 2002; Cardinal and Everitt, 2004). Our finding of a lack of effect of NA lesions crossed with amygdala plus PFo lesions on reinforcer devaluation is consistent with the idea that NA is not critical for goal-directed action; rather, it appears to be critical for some aspects of motivation that promote responding (Cardinal and Everitt, 2004). Our withinsession analysis revealed that the group with crossed disconnection of NA showed a consistent pattern of adaptive choices. Consequently, it seems unlikely that we missed a subtle impairment in this group, one that might have been masked by, for example, learning within the critical sessions. Thus, although neuronal activity in NA reflects the value of expected outcomes based on a history of associated reinforcement (Knutson et al., 2001; Galvan et al., 2005), it is not essential for object choices based on the current value of outcomes.

\section{References}

Aggleton JP, Mishkin M (1983) Memory impairments following restricted medial thalamic lesions in monkeys. Exp Brain Res 52:199-209.

Aggleton JP, Mishkin M (1984) Projections of the amygdala to the thalamus in the cynomolgus monkey. J Comp Neurol 222:56-68.

Baxter MG, Parker A, Lindner CC, Izquierdo AD, Murray EA (2000) Control of response selection by reinforcer value requires interaction of amygdala and orbital prefrontal cortex. J Neurosci 20:4311-4319.

Block AE, Dhanji H, Thompson-Tardif SF, Floresco SB (2007) Thalamicprefrontal cortical-ventral striatal circuitry mediates dissociable components of strategy set shifting. Cereb Cortex 17:1625-1636.

Burke KA, Franz TM, Miller DN, Schoenbaum G (2008) The role of the orbitofrontal cortex in the pursuit of happiness and more specific rewards. Nature 454:340-344.

Cardinal RN, Everitt BJ (2004) Neural and psychological mechanisms underlying appetitive learning: links to drug addiction. Curr Opin Neurobiol 14:156-162.

Chudasama Y, Bussey TJ, Muir JL (2001) Effects of selective thalamic and prelimbic cortex lesions on two types of visual discrimination and reversal learning. Eur J Neurosci 14:1009-1020.

Cooper JC, Knutson B (2008) Valence and salience contribute to nucleus accumbens activation. Neuroimage 39:538-547.

Corbit LH, Muir JL, Balleine BW (2003) Lesions of mediodorsal thalamus and anterior thalamic nuclei produce dissociable effects on instrumental conditioning in rats. Eur J Neurosci 18:1286-1294.

de Wit BR, Rawlins JN, Dickinson A, Balleine BW (2002) Effects of cytotoxic nucleus accumbens lesions on instrumental conditioning in rats. Exp Brain Res 144:50-68.

Floresco SB, Braaksma DN, Phillips AG (1999) Thalamic-cortical-striatal circuitry subserves working memory during delayed responding on a radial arm maze. J Neurosci 19:11061-11071.

Friedman DP, Aggleton JP, Saunders RC (2002) Comparison of hippocampal, amygdala, and perirhinal projections to the nucleus accumbens: combined anterograde and retrograde tracing study in the macaque brain. J Comp Neurol 450:345-365.

Fudge JL, Kunishio K, Walsh P, Richard C, Haber SN (2002) Amygdaloid projections to ventromedial striatal subterritories in the primate. Neuroscience 110:257-275.

Gaffan D, Murray EA (1990) Amygdalar interaction with the mediodorsal nucleus of the thalamus and the ventromedial prefrontal cortex in stimulus-reward associative learning in the monkey. J Neurosci 10:3479-3493.

Gaffan D, Murray EA, Fabrethorpe M (1993) Interaction of the amygdala with the frontal lobe in reward memory. Eur J Neurosci 5:968-975.

Gallagher M, McMahan RW, Schoenbaum G (1999) Orbitofrontal cortex 
and representation of incentive value in associative learning. J Neurosci 19:6610-6614.

Galvan A, Hare TA, Davidson M, Spicer J, Glover G, Casey BJ (2005) The role of ventral frontostriatal circuitry in reward-based learning in humans. J Neurosci 25:8650-8656.

Goldman-Rakic PS, Porrino LJ (1985) The primate mediodorsal (MD) nucleus and its projection to the frontal lobe. J Comp Neurol 242:535-560.

Haber SN, Kunishio K, Mizobuchi M, Lynd-Balta E (1995) The orbital and medial prefrontal circuit through the primate basal ganglia. J Neurosci 15:4851-4867.

Hatfield T, Han JS, Conley M, Gallagher M, Holland P (1996) Neurotoxic lesions of basolateral, but not central, amygdala interfere with Pavlovian second-order conditioning and reinforcer devaluation effects. J Neurosci 16:5256-5265.

Holland PC, Gallagher M (2004) Amygdala-frontal interactions and reward expectancy. Curr Opin Neurobiol 14:148-155.

Hunt PR, Aggleton JP (1991) Medial dorsal thalamic lesions and working memory in the rat. Behav Neural Biol 55:227-246.

Hunt PR, Aggleton JP (1998) Neurotoxic lesions of the dorsomedial thalamus impair the acquisition but not the performance of delayed matching to place by rats: a deficit in shifting response rules. J Neurosci 18:10045-10052.

Izquierdo A, Murray EA (2004) Combined unilateral lesions of the amygdala and orbital prefrontal cortex impair affective processing in rhesus monkeys. J Neurophysiol 91:2023-2039.

Izquierdo A, Murray EA (2007) Selective bilateral amygdala lesions in rhesus monkeys fail to disrupt object reversal learning. J Neurosci 27:10541062.

Izquierdo A, Suda RK, Murray EA (2004) Bilateral orbital prefrontal cortex lesions in rhesus monkeys disrupt choices guided by both reward value and reward contingency. J Neurosci 24:7540-7548.

Johnson AW, Gallagher M, Holland PC (2009) The basolateral amygdala is critical to the expression of Pavlovian and instrumental outcome-specific reinforcer devaluation effects. J Neurosci 29:696-704.

Kennerley SW, Dahmubed AF, Lara AH, Wallis JD (2009) Neurons in the frontal lobe encode the value of multiple decision variables. J Cogn Neurosci 21:1162-1178.

Knutson B, Adams CM, Fong GW, Hommer D (2001) Anticipation of increasing monetary reward selectively recruits nucleus accumbens. J Neurosci 21:RC159.

Knutson B, Rick S, Wimmer GE, Prelec D, Loewenstein G (2007) Neural predictors of purchases. Neuron 53:147-156.

Machado CJ, Bachevalier J (2007) The effects of selective amygdala, orbital frontal cortex or hippocampal formation lesions on reward assessment in nonhuman primates. Eur J Neurosci 25:2885-2904.

Malkova L, Gaffan D, Murray EA (1997) Excitotoxic lesions of the amygdala fail to produce impairment in visual learning for auditory secondary reinforcement but interfere with reinforcer devaluation effects in rhesus monkeys. J Neurosci 17:6011-6020.

McFarland NR, Haber SN (2002) Thalamic relay nuclei of the basal ganglia form both reciprocal and nonreciprocal cortical connections, linking multiple frontal cortical areas. J Neurosci 22:8117-8132.

Mitchell AS, Gaffan D (2008) The magnocellular mediodorsal thalamus is necessary for memory acquisition, but not retrieval. J Neurosci 28:258-263.

Mitchell AS, Browning PG, Baxter MG (2007a) Neurotoxic lesions of the medial mediodorsal nucleus of the thalamus disrupt reinforcer devaluation effects in rhesus monkeys. J Neurosci 27:11289-11295.

Mitchell AS, Baxter MG, Gaffan D (2007b) Dissociable performance on scene learning and strategy implementation after lesions to magnocellular mediodorsal thalamic nucleus. J Neurosci 27:11888-11895.

Neave N, Sahgal A, Aggleton JP (1993) Lack of effect of dorsomedial thalamic lesions on automated tests of spatial memory in the rat. Behav Brain Res 55:39-49.

Ostlund SB, Balleine BW (2008) Differential involvement of the basolateral amygdala and mediodorsal thalamus in instrumental action selection. J Neurosci 28:4398-4405.

Padoa-Schioppa C, Assad JA (2006) Neurons in the orbitofrontal cortex encode economic value. Nature 441:223-226.

Peciña S, Berridge KC (2005) Hedonic hot spot in nucleus accumbens shell: where do $\mu$-opioids cause increased hedonic impact of sweetness? J Neurosci 25:11777-11786.

Pickens CL (2008) A limited role for mediodorsal thalamus in devaluation tasks. Behav Neurosci 122:659-676.

Pickens CL, Saddoris MP, Setlow B, Gallagher M, Holland PC, Schoenbaum G (2003) Different roles for orbitofrontal cortex and basolateral amygdala in a reinforcer devaluation task. J Neurosci 23:11078-11084.

Preuss TM, Goldman-Rakic PS (1987) Crossed corticothalamic and thalamocortical connections of macaque prefrontal cortex. J Comp Neurol 257:269-281.

Ray JP, Price JL (1993) The organization of projections from the mediodorsal nucleus of the thalamus to orbital and medial prefrontal cortex in macaque monkeys. J Comp Neurol 337:1-31.

Rose JE, Woolsey CN (1943) A study of thalamocortical relations in the rabbit. Bull Johns Hopkins Hosp 73:65-128.

Rudebeck PH, Behrens TE, Kennerley SW, Baxter MG, Buckley MJ, Walton ME, Rushworth MF (2008) Frontal cortex subregions play distinct roles in choices between actions and stimuli. J Neurosci 28:13775-13785.

Selemon LD, Goldman-Rakic PS (1985) Longitudinal topography and interdigitation of corticostriatal projections in the rhesus monkey. J Neurosci 5:776-794.

Stern CE, Passingham RE (1996) The nucleus accumbens in monkeys (Macaca fascicularis): II. Emotion and motivation. Behav Brain Res 75:179-193.

Walker AE (1940) A cytoarchitectonic study of the prefrontal area of the macaque monkey. J Comp Neurol 73:59-86.

Wallis JD, Miller EK (2003) Neuronal activity in primate dorsolateral and orbital prefrontal cortex during performance of a reward preference task. Eur J Neurosci 18:2069-2081.

Wang SH, Ostlund SB, Nader K, Balleine BW (2005) Consolidation and reconsolidation of incentive learning in the amygdala. J Neurosci 25: $830-835$.

Wellman LL, Gale K, Malkova L (2005) GABAA-mediated inhibition of basolateral amygdala blocks reward devaluation in macaques. J Neurosci 25:4577-4586. 